\title{
Síndrome mascarada como primeira manifestação de adenocarcinoma pulmonar - Relato de caso e revisão de literatura
}

\section{Masquerade syndrome as the first manifestation oflung adenocarcinoma-Case report}

\author{
Charles Costa de Farias ${ }^{1}$ \\ Claudio Renato Garcia ${ }^{2}$ \\ Cristina Muccioli ${ }^{3}$
}

\section{RESUMO}

Objetivo: Os autores relatam um caso de paciente com síndrome mascarada como primeira manifestação de adenocarcinoma pulmonar. Métodos: $\mathrm{Pa}$ ciente de 57 anos com história clínica de embaçamento visual unilateral (olho direito) de seis meses de evolução e diagnóstico inicial de uveíte recidivante com tratamento à base de corticóide tópico. Referida por permanência dos sintomas mesmo com tratamento. Ao exame ocular apresentava hiperemia conjuntival com injeção ciliar moderada e lesão peripapilar na coróide. Solicitados os exames complementares. Resultados: A paciente foi diagnosticada com adenocarcinoma pulmonar (lesão primária). As alterações em fundo de olho foram sugestivas de lesões metastáticas na coróide. Conclusões: A incidência das metástases uveais varia de 0,7 a $12 \%$ dos pacientes com tumores pulmonares. $\mathrm{O}$ tratamento depende de características como tamanho da lesão, número de metástases, acuidade visual, bilateralidade e estado geral do paciente. O envolvimento ocular pode ser a primeira manifestação clínica de adenocarcinoma pulmonar, conforme observado neste caso.

Descritores: Uveíte/complicações; Neoplasias pulmonares/complicações; Adenocarcinoma; Neoplasias da coróide/secundária; Metástase neoplásica; Relato de caso

Trabalho realizado no Setor de Uveítes e AIDS do Departamento de Oftalmologia da Universidade Federal de São Paulo, Escola Paulista de Medicina UNIFESP/EPM.

${ }^{1}$ Estagiário do Setor de Uveítes e AIDS do Departamento de Oftalmologia da Universidade Federal de São Paulo, Escola Paulista de Medicina - UNIFESP/EPM. 2 Pós-graduando nível Doutorado do Setor de Uveítes e AIDS do Departamento de Oftalmologia da Universidade Federal de São Paulo, Escola Paulista de Medicina - UNIFESP/EPM.

${ }^{3}$ Professora afiliada e Chefe do Setor de Uveítes e AIDS do Departamento de Oftalmologia da Universidade Federal de São Paulo, Escola Paulista de Medicina UNIFESP/EPM.

Endereço para correspondência: Rua Botucatu, 822 São Paulo (SP) CEP 04023-062

E-mail: Charles-costa@ uol.com.br

Recebido para publicação em 16.01.2002 Aceito para publicação em 12.06.2002

Nota Editorial: Pela análise deste trabalho e por sua anuência na divulgação desta nota, agradecemos ao $\mathrm{Dr}$. Haroldo Vieira de Moraes Junior.

\section{INTRODUÇ̃̃̃O}

Os tumores oculares malignos mais freqüentes são os carcinomas metastáticos ${ }^{(1-2)}$. Os primeiros casos descritos foram relatados por Horne ${ }^{(3)} \mathrm{e}$ $\operatorname{Perl}^{(4)}$ e considerados naquela época diagnósticos infreqüentes. Os melanomas malignos de coróide são historicamente descritos como sendo os tumores malignos intra-oculares mais comuns em adultos. Dados estatísticos sugerem que a ocorrência de metástases para o olho, órbita ou ambos é de aproximadamente $12 \%{ }^{(5)}$. A região ocular mais acometida pelo tumor metastático é o trato uveal ${ }^{(6-8)}$. Menos freqüentemente as metástases podem acometer a pálpebra ${ }^{(9-10)}$, conjuntiva ${ }^{(11-12)}$, nervo óptico ${ }^{(6)}$ ou órbita ${ }^{(13-14)}$.

Aproximadamente $22 \%$ de todas as metástases intra-oculares em homens e mulheres assim como $44 \%$ das metástases intra-oculares em homens são secundárias a tumores pulmonares; enquanto a incidência geral varia em torno de 5 a $6,7 \%{ }^{(5,15)}$.

A incidência real dos tumores metastáticos oculares não é determinada de forma absoluta, já que alguns pacientes não procuram avaliação oftalmológica. $\mathrm{O}$ aumento na incidência dos tumores metastáticos que acometem a úvea e globo ocular em geral guarda relação direta com a expectativa de
\end{abstract}


vida. Os tratamentos com radioterapia e/ou quimioterapia desempenham papel fundamental nas estatísticas destas metástases.

\section{RELATO DE CASO}

Paciente do sexo feminino de 57 anos de idade procedente de São Paulo com história de embaçamento visual e hiperemia no olho direito há 6 meses e com diagnóstico de uveíte posterior crônica córtico-dependente e recidivante. Na ocasião fazia uso de corticóide tópico a cada 2 horas e referia piora dos sintomas.

A paciente havia sido tratada para toxoplasmose com Prednisona $80 \mathrm{mg} / \mathrm{dia}$, Sulfametoxazol 800mg+Trimetropim160mg/ dia, Atropina e Dexametasona colírio e referia ter apresentado três recidivas oculares em 2 meses.

Ao exame clínico a paciente apresentava acuidade visual de 20/100 OD e 20/20 OE, olho esquerdo apresentava exame oftalmológico normal e no olho direito foram observadas hiperemia conjuntival discreta com injeção ciliar moderada, córnea transparente, ausência de reação de câmara anterior, aumento da refringência do cristalino, $2+$ de células vítreas, edema de papila, hemorragia retiniana peripapilar e lesão esbranquiçada elevada em pólo posterior localizada na arcada nasal superior próxima ao nervo óptico (Figura 1).

Os exames laboratoriais mostravam hemograma normal, exame de urina normal, hemossedimentação discretamente aumentada, PPD negativo, sorologia para toxoplasmose IgM negativo IgG 1=169, hemaglutinação de 1/500, VDRL e FTAbs negativos. A angiografia fluoresceínica mostrava lesão ativa tipo coriorretinite no OD (Figura 2). Não foram observadas alterações no $\mathrm{OE}$.

Com base na história e exame clínico da paciente novos exames foram solicitados. $\mathrm{O}$ exame radiológico revelou opacidade tênue de contornos parcialmente bem definidos em projeção basal direita. Foram solicitadas tomografia axial computadorizada de tórax, espirometria, dosagem de enzima conversora de angiotensina (ECA) e avaliação por pneumologista. A

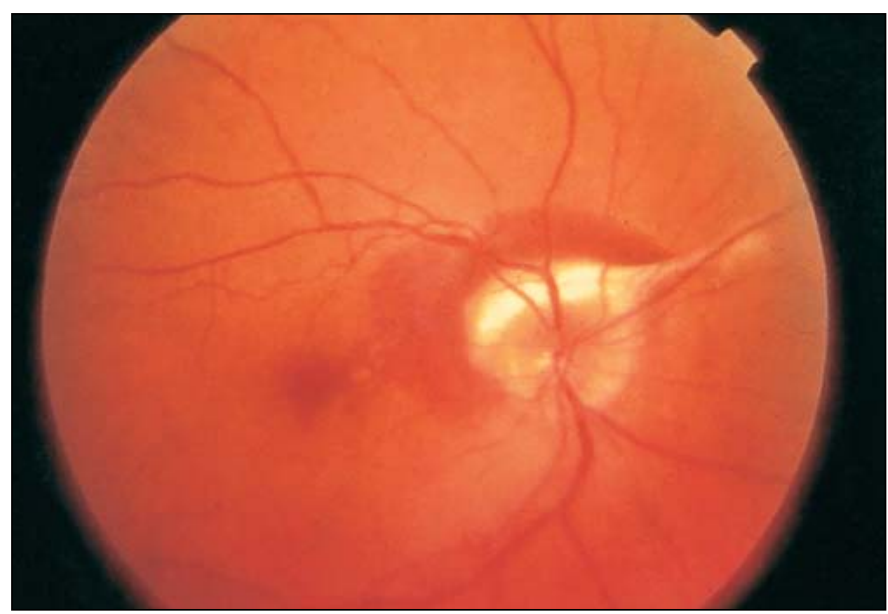

Figura 1 - Edema de papila, hemorragia retiniana peripapilar e lesão esbranquiçada no pólo posterior tomografia axial computadorizada revelou massa pulmonar à direita com vaso calibroso drenando a mesma e adenomegalias mediastinais. A espirometria revelou volume de reserva expiratório acentuadamente diminuído sem alterações no exame com broncodilatador. Os resultados de exames solicitados revelaram ASLO negativo e ECA de 7,5U/1.

No momento da avaliação por pneumologista a paciente evoluiu com episódios de piora da AV e apresentava 4+ de células vítreas e melhora do edema de papila, diminuição da hemorragia sub-retiniana macular e exsudatos duros maculares próximos da papila. Diante do quadro apresentado foi prescrito a diminuição progressiva de Prednisona para 40mg/dia.

Foi realizada biópsia pulmonar diagnóstica e o exame histopatológico revelou adenocarcinoma pouco diferenciado com comprometimento linfonodal. Radioterapia e quimioterapia foram realizadas para tratamento da condição sistêmica. Pesquisa de metástases ósseas foi feita com cintilografia óssea e foi negativa.

A paciente evoluiu com períodos de remissão e recidiva do quadro ocular (Figura 3) e mesmo tratada para condição sistêmica evolui para óbito em 6 meses após diagnóstico.

\section{DISCUSS ÃO}

A neoplasia ocular mais freqüente em adultos é de origem metastática $^{(16-17)}$. No homem o pulmão é o foco de disseminação mais freqüente da lesão primária e a mama nas mulheres. $\mathrm{O}$ diagnóstico da lesão inicial ocorre em média 2 a 3 anos após a metástase ocular ${ }^{(18)}$.

As metástases oculares citadas acima tem sua maior incidência entre a quinta e sétima década de vida ${ }^{(17)}$. A úvea é o principal local de metástases, devido a sua rica rede vascular e a coróide é geralmente o local mais acometido ${ }^{(18-19)}$.

A baixa da acuidade visual constitui-se no sintoma mais freqüente de metástase ocular, devido a propagação do tumor até a mácula ou descolamento seroso da retina. A perda da

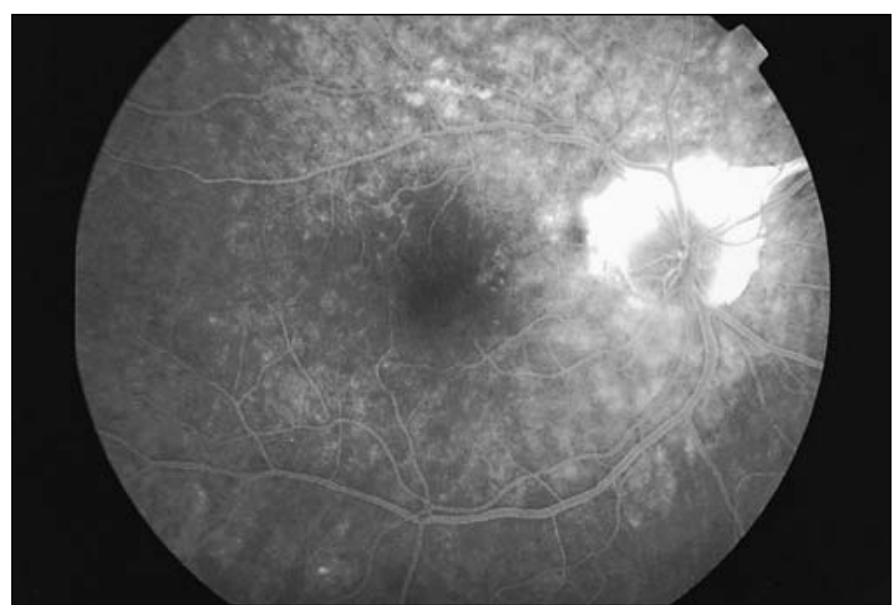

Figura 2 - Presença de área de hiperfluorescência com "leakage" tardio 


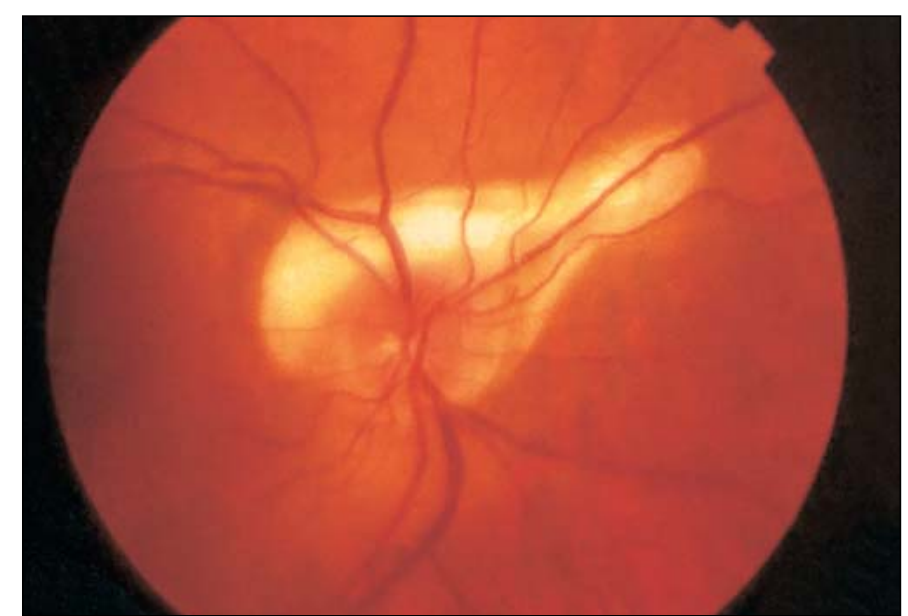

Figura 3 - Diminuição do edema e hiperemia de papila, ausência de hemorragia

visão pode vir acompanhada de outros sintomas como diplopia, edema periorbital, oftalmoplegia, dor, glaucoma, proptose, presença de lesões amarelo-creme multifocais localizadas na coróide, fotopsia, exoftalmia, papiledema, uveítes, descolamento de retina e descolamento de coróide ${ }^{(16,18,20-22)}$.

A descoberta precoce do câncer de pulmão é uma tarefa difícil, já que os sintomas sistêmicos iniciais podem ser de apresentação subclínica. Em condições crônicas o paciente desenvolve dispnéia, hemoptise, tosse, perda de peso, pneumonites, dor torácica, respiração ruidosa e rouquidão ${ }^{(23)}$.

$\mathrm{O}$ fator determinante mais importante que orienta para diagnóstico de tumor metástico é a história positiva de câncer em qualquer parte do corpo antes do aparecimento de lesão ocu$\operatorname{lar}^{(24-25)}$.

Os exames laboratoriais podem ser úteis em orientar se a lesão ocular é um tumor primário ou metastático.

O tratamento do paciente com metástase depende da localização do tumor primário e lesão ocular, tipo de células e condições gerais do paciente.

A observação é recomendada quando o paciente tem comprometimento monocular, com diagnóstico inconclusivo, idosos ou com estado geral comprometido.

A quimioterapia é indicada para metástases sistêmicas sem a presença de metástases coroidais. Geralmente é ineficaz e menos responsiva do que radioterapia.

A radioterapia está indicada nos casos de metástases uveais múltiplas ou únicas resistentes à quimioterapia, ou quando os tumores sistêmicos e de coróide são progressivos.

A enucleação reserva-se para olhos com dor intratável, glaucoma secundário ou cegueira. Injeção retrobulbar de álcool pode ser uma opção no caso de dor orbital, sem necessidade de enucleação ${ }^{(26-28)}$.

\section{CONCLUSÃO}

Um sintoma visual inespecífico ou um achado de fundo de olho especialmente de coróide pode ser o primeiro indício de câncer, principalmente para certos tipos tais como o câncer de pulmão semelhante ao caso relatado. Uma avaliação precisa destes pacientes com o objetivo de se detectar o mais precocemente possível as alterações compatíveis com lesão tumoral metastática e encaminhar para especialista a fim de se instituir terapêutica adequada torna-se uma necessidade.

\section{ABSTRACT}

Purpose: To describe a case of masquerade syndrome as the first manifestation of lung adenocarcinoma. Methods: A 57year-old female was referred with a 6-month history of blurred vision in her right eye. She was treated for relapsing uveitis but signs and symptons remained despite treatment with topical steroids. On physical examination she had conjuctival hyperemia, moderate cilliary injection and choroidal peripapillary lesion. Ancillary examinations were requested. Results: A lung adenocacirnoma was diagnosed as the primary lesion. The choroidal peripapillary lesion in her right eye was consistent with the appearance of a choroidal metastatic lesion. Conclusions: The incidence of uveal metastasis ranges from 0.7 to $12 \%$ in patients with pulmonary tumors. Treatment depends on characteristics such as size of the lesion, number of metastases, visual acuity, bilaterality and the patient's general health. As confirmed by this case, ocular involvement may be the first clinical manifestation of lung adenocarcinoma.

Keywords: Lung neoplasms/complications; Adenocarcinoma; Choroid neoplasms/secondary; Neoplasm metastasis; Case report

\section{REFERÊNCIAS}

1. Oliveira AA, Oréfice F, Miranda D. Carcinoma metastático na coróide simulando doença de Harada. Arq Bras Oftalmol 1983;46:31-5.

2. Cialdini A, Ávila M. Indocianina verde em tumores de coróide. Arq Bras Oftalmol 1998;61:210-22.

3. Horner F. Carcinoma der dura mater. Exophthalmus. Carcinoma der musculi recti. Allyeime carcinose. Lin Monatsble Augenheilkd 1862;2:186-90.

4. Perl M. Contribution to pathology of tumor. Virchow Arch [A]1872;56:437-67.

5. Bloch RS, Gartner S. The incidence of ocular metastatic carcinoma. Arch Ophthalmol 1971;85:673-5.

6. Shields JA, Shields CL. Metastatic tumor to the intraocular structures. In: Shields JA, Shields CS. Intraocular tumors: a text and atlas. Philadelphia: W.B.Saunders; 1992. p.207-38.

7. Shields JA, Shields CL, Kiratli H, De Potter P. Metastatic tumors to the iris in 40 patients. Am J Ophthalmol 1995;119:422-30.

8. Shields CL, Shields JA, Gross N, Schwartz G, Lally S. Survey of 520 uveal metastases. Ophthalmology 1997;104:1265-76.

9. Riley FC. Metastatic tumors to the eyelids. Am J Ophthalmol. 1970;69;259-64.

10. Mansour AM, Hidayat AA. Metastatic eyelid disease. Ophthalmology 1987; 94:667-70

11. Kiratli H, Shields CL, Shields JA, De Potter P. Metastatic tumors to the conjunctiva [commented on Br J Ophthalmol 1996;80:1]. Br J Ophthalmol 1996;80:5-8.

12. Shields JA, Gunduz K, Shields CL, Eagle RC Jr, De Potter P, Van Rens E. Conjuntival metastasis as the initial manifestations of lung cancer. Am J Ophthalmol 1997;124:399-400. 
13. Shields CL, Shields JA, Peggs M. Tumors metastatic to the orbit. Ophthal Plast Reconstr Surg 1988;42:73-80.

14. Shields JA. Metastatic cancer to the orbit. In: Shieldes JA. Diagnosis and management of orbital tumors. Philadelphia: W.B. Saunders; 1989. p.291315 .

15. Nelson CC, Hertzberg BS, Klintworth GK. A histopathologic study of 716 unselected eyes in patients with cancer at the time of death. Am J Ophthalmol 1983;95:788-93.

16. Ferry AP, Font RL. Carcinoma metastatic to the eye and orbit. I. A clinicopathologic study of 227 cases. Arch Ophthalmol 1974;92:276- 86.

17. Ferry AP. The biological behavior and pathological features of carcinoma metastatic to the eye and orbit. Trans Am Ophthalmol Soc 1973;71:373-425.

18. Stephens RF, Shields JA. Diagnosis and management of cancer metastatic to the uvea: a study of 70 cases. Ophthalmology 1977;86:1336.

19. Shields JA, Young SE. Malignant tumors of the uveal tract. Curr Probl Cancer 1980;5:1-35.

20. Swanson MW. Metastatic tumor formation: processes within visual system. J Am Optom Assoc 1990;61:269-308.

21. White BR, Prouty RE. Presenting symptomatology of diplopia results in a diagnosis of metastatic breast cancer: a case report. South J Optom 1994; $8: 12-8$.

22. Sneed SR, Byrne SF, Mieler WF, Nicholson DH, Olsen K, Hughes JR. Choroidal detachment associated with malignant choroidal tumors. Ophthalmology 1991;98:963-70.

23. Minna J, Higgins GA, Glatstein EJ. Cancer of the lung. In: De Vitta Jr VT, Hellman S, Rosemberg SA. editors. Principles and pratice of oncology. Philadelphia: Lippincott; 1981. p.517-24.

24. Hart WM. Metastatic carcioma of the eye and orbit. Int Ophthalmol Clin 1962;2:465-82.

25. Michelson JB, Stephens RF, Shields JA. Clinical conditions mistaken for metastatic cancer to the choroid. Am Ophthalmol 1979;11:140-53.

26. Char DH. Posterior uveal tumors. In: Char DH, editor. Clinical ocular oncology. New York: Churchill Livingstone; 1989. p.91-149.

27. Burmeisteir BH, Benjamin CS, Childs WJ. The management of metastases to eye and orbit from carcinoma of the breast. Aust N Z J Ophthalmol 1990,18:187-90.

28. Nylen U, Kock E, Lax I, Lundell G, Af Trampe E, Wilking N. Standardized precision theraphy choroidal metastases. Acta Oncol 1994;33:65-8.

\title{
$2^{\circ}$ CONGRESSO BRASILEIRO DE CATARATA E CIRURGIA REFRATIVA
}

\author{
14 a 18 de Maio de 2003 \\ SOFITEL CONVENTIONS \& RESORT \\ costa do sAUÍPE - BAHIA
}

\section{Promoção}

- Sociedade Brasileira de Catarata e Implantes Intra-Oculares

- Sociedade Brasileira de Cirurgia Refrativa

\author{
INFORMAC̣ÕES: JDE Comunicação e Eventos \\ Tels.: (1 1) 287-8109/287-9699/ 287-9378 \\ Fax: (11) 288-8157 \\ E-mail: jdecomev@uol.com.br
}

